\title{
Novel Adaptive PID Control of Flexible Joint Robot Manipulator withBounded Disturbances
}

\author{
Hossam N. Doghiem* \\ Design and Production Engineering Department,Faculty of Engineering, Ain Shams University, Cairo, Egypt
}

\begin{abstract}
A novel robust adaptive PID control scheme is proposed with known upper bound of the external disturbances, to solve the dynamic coupling and strong nonlinearity problems in flexible joint robot manipulator control. Invoking the proposed controller, the bounded external disturbances can be compensated and the global asymptotical stability with respect to the manipulator positions and velocities is able to be guaranteed. The designed control law can enlarge the tolerable external disturbances, enhance the accuracy of trajectory tracking error, and improve the dynamic performance of the manipulator systems. The stability and convergence properties of the closed-loop system are analytically proved using Lyapunov stability theory and Barbalat's lemma. Simulation for the proposed control system is performed for a two-degree of freedom flexible joint robot, each joint modeled by two-equations of second order to illustrate its viability, and advantages.
\end{abstract}

Keywords: - Adaptive PID controller, Dynamic coupling, Flexible joint robot, Bounded external disturbances, Lyapunov stability theory, Trajectory tracking error.

\section{INTRODUCTION}

Robot manipulators play an important part in modern industry by providing lower production cost, enhanced precision, quality, productivity, and efficiency. The control of robot faces significant difficulties, with regard to such a complicated system, various controllers have been developed [1], such as adaptive controllers [2-4], robust controllers [5-7] and controllers based on the theory of variable structure [8-11]. However, the applicability of these controllers to practical robot is limited because the assumption of perfect rigidity is never satisfied exactly.

Since linear control methods are not suitable for strong coupled, nonlinear and time-varying flexible robot manipulator systems, many nonlinear control schemes based on conventional PID control theory have been proposed to improve the control performance. In [12], the global asymptotic stability of a class of nonlinear PD-type controllers for position and motion control of robot manipulators is analyzed, and a global regulator constrained to deliver torques within prescribed limits of the actuator's capabilities is proposed.

However, it has been shown that although the PD controller is robust with respect to uncertainties on inertial parameters and the global asymptotic stability is guaranteed, uncertainties on the gravity parameters may lead to undesired steady state errors [13]. A PID control scheme can eliminate the steady-state errors, but it can only ensure local asymptotic stability. Moreover, to guarantee the stability, the gain matrices must satisfy complicated inequalities [14]. In [15], a new variable structure PID control scheme is designed for robot manipulators.

Robust control laws are used for external disturbances, unstructured dynamics, and other sources of uncertainties. [16,17] present a popular approach for designing robust controller. A simple robust nonlinear control law is derived by [18] using the approach of [17] for $\mathrm{n}$-link robot manipulators using the well-known Lyapunov theory to guarantee stability of uncertain systems. Other control methods developed based on $[16,17]$ are given in $[19,20]$. However, disturbances and unmodeled dynamics are not considered in the algorithms in [18-20]. In [21], the method developed by [18] is modified such that the controller is robust to unmodeled dynamics and disturbances.

Most adaptive algorithms may exhibit poor robustness to unstructured dynamics and external disturbances, to resolve this, a combination of robust control and adaptive algorithm is investigated in a number of literatures. In[22], adaptive controllers are designed for robot manipulators that yield robust trajectory in spite of the unwanted effects of the external disturbances and fast maneuvering of the manipulator. The algorithm presented in [23] is suitable for swift adaptation to rapidly changing system parameters. In [24], a decentralized adaptive controller is investigated for trajectory tracking of robot manipulator systems. A disturbance observer is introduced in each controller to compensate for coupled uncertainties, and an adaptive sliding mode control term is employed to handle the fast changing components of the uncertainties beyond the pass band of the disturbance observer.

A novel robust decentralized control scheme by adaptive fuzzy estimation and compensation uncertainty is proposed by [25]. The controller is designed via voltage control strategy, a fuzzy system is used to 
estimate and compensate uncertainty. In [26,27], two adaptive PD control methods are investigated with known and unknown upper bound of the external disturbances, respectively. Both of the controllers are composed by a nonlinear PD feedback control law and an adaptive algorithm. However, the PD feedback control is rarely used because the differential action is very sensitive to system noise. Hence, the PD control is of no benefit for the improvement of the system dynamic performance.

Robust tracking controller for (FJR) is developed using voltage control strategy [28], achieving pre-set performance on link position error [29] both are free of manipulator dynamics and nonlinearities. Adaptive trajectory control scheme consists of a direct (MRAS) is presented [30] to improve damping of vibration at the joints. An adaptive fussy output feedback approach is proposed [31] to compensate for nonlinear dynamics while only requiring the measurement of link position.

In the present study a new robust adaptive PID controller is introduced for an $\mathrm{n}$ degree-of-freedom robot manipulator systems with known upper bound of the external disturbances based on [12,26,27]. The designed controller is composited by PID control and robust adaptive approach to cope with the external disturbances and unknown constant parameters that can arise. An integration element is embedded in both PD control and robust adaptive algorithm based on the existing adaptive PD control law [26,27]. With the adoption of the proposed controller, the tolerable external disturbances are enlarged, and also the dynamic performance of the system is improved. By choosing adequate Lyapunov candidate functions and utilizing Barbalat's lemma, the system's closed-loop stability is proven. Same numerical simulation studies are carried out in order to demonstrate the control system performance.

\section{DYNAMIC MODEL FOR (FJR)}

This section describes the dynamic model of the robot. This model is used for the design and control development. The dynamic model for flexible joint robot developed by [32] is adopted. It is derived for the experimental robot using Euler-Lagrange equation [33], and it is given by the following equations:

$\mathrm{M}(\mathrm{q}) \ddot{q}+\mathrm{C}(\mathrm{q}, \dot{q})+\mathrm{B} \dot{q}-\mathrm{K}\left(\mathrm{q}_{\mathrm{m}}-\mathrm{q}\right)+\mathrm{G}(\mathrm{q})=-\mathrm{J}^{\mathrm{T}} \quad \mathrm{F}$

$\mathrm{I}_{\mathrm{m}} \ddot{q}_{\mathrm{m}}+\mathrm{B}_{\mathrm{m}} \dot{q}_{\mathrm{m}}+\mathrm{K}\left(\mathrm{q}_{\mathrm{m}}-\mathrm{q}\right)=\tau$

Where $\mathrm{q}$ is the $2 \times 1$ link angular position vector, $\mathrm{q}_{\mathrm{m}}$ is the $2 \times 1$ motor angular position vector, $\mathrm{M}(\mathrm{q})$ is the $2 \times 2$ manipulator inertia matrix, $\mathrm{C}(\mathrm{q}, \dot{q})$ is the $2 \mathrm{x} 1$ coriolos and centrifugal forces vector, $\mathrm{K}$ is $2 \times 2$ diagonal matrix with entries equal to the joint stiffness, $\mathrm{G}(\mathrm{q})$ is the $2 \mathrm{x} 1$ gravity force vector, $\mathrm{J}^{\mathrm{T}}$ is the $2 \times 2$ transpose of the manipulator Jacobian, $F$ is $2 \times 1$ forces vector at the end effector expressed in the reference frame, $I_{m}$ is the $2 \times 2$ diagonal matrix with entries equal to the rotors inertia, $B_{m}$ is the $2 \times 2$ diagonal matrix with entries equal to the coefficient of viscous damping at the motors, and $\tau$ is $2 \times 1$ applied motor torque vector.

\section{Problem Formulation}

The process of controlling the dynamic model given by equations (1) is difficult because the system is multi-input multi-output (MIMO) nonlinear. However, considering each link and its driving motor only reduces the system to two single input multi-output linear subsystems, whichsimplifies the identification and control process. [34] Has implemented this identification technique on a two-link flexible joint experimental robot. The first subsystem is the first joint (the first motor and the first link) and the second subsystem is the second joint (the second motor and the second link). The following procedures are performed to control these two subsystems.

a) First, constrain the first subsystem by clamping the first link to the fixed table, and thus the second subsystem characteristic can be isolated, identified, and control.

b) To identify and control the first subsystem, the brake of the second motor is applied. Hence, the second subsystem is considered as extra mass add to the end of the first link.

Dynamic System Properties: The dynamic systems given by (1) exhibit the following properties that are utilized in the subsequent control law development and stability analysis [33].

a) (P1) The inertial matrix is symmetric and positive definite; that is, $\mathrm{M}(\mathrm{q})=\mathrm{M}^{\mathrm{T}}(\mathrm{q})>0 \forall q \in R^{n}$. There are positive constants $\mathrm{m}_{\mathrm{m}}$ and $\mathrm{M}_{\mathrm{m}}$ such that $m_{m}\|y\|^{2} \leq y^{T} M(q) y \leq m_{M}\|y\|^{2}, \forall y \in R^{n}$.

b) $(\mathrm{P} 2) \dot{M}(q)-2 C(q, \dot{q})$ is a skew-symmetric matrix; for example, $s^{T}[\dot{M}(q)-2 C(q, \dot{q})] s=0, \forall s \in R^{n}$.

c) $(\mathrm{P} 3) \mathrm{M}(\mathrm{q}), \mathrm{C}(\mathrm{q}, \dot{q})$, and $\mathrm{G}(\mathrm{q})$ meet the linear condition of,

$\mathrm{M}(\mathrm{q}) \propto+C(q, \dot{q}) \beta+G(q)=\psi(q, \dot{q}, \alpha, \beta) P$, where $\mathrm{P} \in R^{m}$ is an unknown constant vector which describes the mass characteristics of the manipulator and $\psi(q, \dot{q}, \alpha, \beta) \in R^{n x m}$ is a known regression matrix.

\section{ROBUST ADAPTIVE PID CONTROL OF ROBOT}

Firstly, the following assumptions are imposed for the manipulator systems.

1. (A1) The desired trajectory $q_{d}$ and the time derivatives $\dot{q}_{d a n d} \ddot{q}_{d}$ are available and bounded signals.

2. (A2) The external disturbances vector $u$ is bounded, and it is confined within the following limit: 
$\|u\| \leq b_{1}+b_{2}\|e\|+b_{3}\|\dot{e}\|+b_{4}\left\|\int_{0}^{t} e d t\right\|$ Where,

$\mathrm{b}_{1}, \mathrm{~b}_{2}, \mathrm{~b}_{3}$, and $\mathrm{b}_{4}$ are positive constants, $\mathrm{e}=\mathrm{q}-q_{d}$ and $\dot{e}=\dot{q}-\dot{q}_{d}$ are the position tracking error and the velocity tracking error, respectively, $\int_{0}^{t} e_{1} d t=\left[\int_{0}^{t} e_{1} d t, \ldots . . \int_{0}^{t} e_{n} d t\right]^{\mathrm{T}}$.

3.(A3) $\ddot{e}=\ddot{q}-\ddot{q}_{d}$ is existent and bounded in t. Here we introduce two variables $\mathrm{x}$ and $q_{k}$; meanwhile let

$x=\dot{e}+\gamma e+\int_{0}^{t} e d t, \dot{q}_{k}=\dot{q}_{d}-\gamma e-\int_{0}^{t} e d t$,

Where the parameter $\gamma$ is a positive constant, with (3) giving

$\dot{q}-\dot{q}_{k}=x$

With regard to robot manipulator property (P3), let $=\ddot{q}_{k}, \beta=\dot{q}_{k}$; one obtains

$M(q) \ddot{q}_{k}+C(q, \dot{q}) \dot{q}_{k}+G(q)=\Psi\left(q, \dot{q}, \dot{q}_{k}, \ddot{q}_{k}\right) P$

Substituting Eqn. (4) into Eqn. (5) yields

$M(q) \ddot{q}+C(q, \dot{q}) \dot{q}+G(q)-M(q) \dot{x}-C(q, \dot{q}) x=\Psi\left(q, \dot{q}, \dot{q}_{k}, \ddot{q}_{k}\right) P$

\section{DESIGN OF ROBUST ADAPTIVE PID CONTROLLER}

For the robot manipulator systems (1), if the upper bound of the external disturbances signals $u$ is known, motivated by $[12,26]$, the controller which makes the position and the velocity tracking errors asymptotically converge to zero can be designed as follows:

$$
\begin{aligned}
& \tau=-K_{P} e-K_{D} \dot{e}-K_{I}\left(\int_{0}^{t} e d t\right)+\Psi\left(q, \dot{q}, \dot{q}_{k}, \ddot{q}_{k}\right) \hat{p}+V \\
& V=\left[v_{1}, \ldots ., v_{n}\right]^{\mathrm{T}} \\
& v_{i}=-\left(b_{1}+b_{2}\|e\|+b_{3}\|\dot{e}\|+b_{4}\left\|\int_{0}^{t} e d t\right\|\right) \operatorname{sgn}\left(x_{i}\right)
\end{aligned}
$$

Where $\hat{P}$ is the estimate value of $\mathrm{P}$. Take the parameter estimation law of $\hat{P}$ as

$\dot{\hat{P}}=-\Phi \Psi^{T}\left(q, \dot{q}, \dot{q}_{k}, \ddot{q}_{k}\right) x$

The gain matrices are given by

$$
\begin{aligned}
K_{P} & =\operatorname{diag}\left[K_{P 1}, \ldots ., K_{P n}\right], \\
K_{D} & =\operatorname{diag}\left[K_{D 1}, \ldots, K_{D n}\right] \\
& K_{I}=\operatorname{diag}\left[K_{I 1}, \ldots, K_{I n}\right]
\end{aligned}
$$

Where $K_{P i}, K_{D i}, K_{I i}(\mathrm{I}=1,2, \ldots, \mathrm{n})$ are all positive constants and $K_{D i}=K_{I i}, \Phi \in R^{m \times m}$ is a positive definite and symmetric matrix. The framework of the proposed control scheme is shown in Fig. 1.

Proof: Considering the Lyapunov function candidate,

$V=\frac{1}{2}\left[x^{T} M(q) x+e^{T}\left(K_{P}+\gamma K_{D}\right) e+\left(\int_{0}^{t} e d t\right)^{T}\left(K_{P}+\gamma K_{I}\right)\left(\int_{0}^{t} e d t\right)+\tilde{P}^{T} \Phi^{-1} \tilde{P}\right]$

With $\tilde{P}=\hat{P}-P$, from property (P1) one obtains

$\left(x^{T} M(q) x\right)^{\prime}=\dot{x}^{T} M(q) x+x^{T} \dot{M}(q) x+x^{T} M(q) \dot{x}=x^{T} \dot{M}(q) x+2 x^{T} M(q) \dot{x}$.

With the positive definite and symmetric matrices $K_{P}, K_{D}, K_{I}$, and $\Phi$, onegets

$$
\left[e^{T}\left(K_{P}+\gamma K_{D}\right) e\right]^{\prime}=2 e^{T}\left(K_{P}+\gamma K_{D}\right) \dot{e},
$$

$\left[\left(\int_{0}^{t} e d t\right)^{T}\left(K_{P}+\gamma K_{I}\right)\left(\int_{0}^{t} e d t\right)\right]^{\prime}=2\left(\int_{0}^{t} e d t\right)^{T}\left(K_{P}+\gamma K_{I}\right) e$,

Therefore, one obtains

$$
\left(\tilde{P}^{T} \Phi^{-1} \tilde{P}\right)^{\prime}=2 \tilde{P}^{T} \Phi^{-1} \dot{\tilde{P}}
$$

$\dot{V}=1 / 2 x^{T} \dot{M}(q) x+x^{T} M(q) \dot{x}+e^{T}\left(K_{P}+\gamma K_{D}\right) \dot{e}+\left(\int_{0}^{t} e d t\right)^{T}\left(K_{P}+\gamma K_{I}\right) e+\tilde{P}^{T} \Phi^{-1} \dot{\widetilde{P}}$

Using (6) and (7) leads to

$$
\begin{aligned}
& x^{T} M(q) \dot{x}=x^{T}\left[\tau-u-\Psi\left(q, \dot{q}, \dot{q}_{k}, \ddot{q}_{k}\right) P-C(q, \dot{q}) x\right]= \\
& x^{T}\left[-K_{P} e-K_{D} \dot{e}-K_{I}\left(\int_{0}^{t} e d t\right)+\Psi\left(q, \dot{q}, \dot{q}_{k}, \ddot{q}_{k}\right) \tilde{P}+V-u\right]-x^{T} C(q, \dot{q}) x .
\end{aligned}
$$

Considering $x^{T}=\dot{e}^{T}+\gamma e^{T}+\left(\int_{0}^{t} e d t\right)^{T}$, one gets

$$
x^{T}\left(-K_{P} e-K_{D} \dot{e}-K_{I} \int_{0}^{t} e d t\right)=\left[\dot{e}^{T}+\gamma e^{T}+\left(\int_{0}^{t} e d t\right)^{T}\right]\left[K_{P} e-K_{D} \dot{e}-K_{I}\left(\int_{0}^{t} e d t\right)\right]
$$




$$
\begin{aligned}
& =-\dot{e}^{T} K_{P} e-\dot{e}^{T} K_{D} \dot{e}-\dot{e}^{T} K_{I}\left(\int_{0}^{t} e d t\right)-\gamma e^{T} K_{P} e-\gamma e^{T} K_{D} \dot{e}-\gamma e^{T} K_{I}\left(\int_{0}^{t} e d t\right)-\left(\int_{0}^{t} e d t\right)^{T} K_{P} e- \\
& \left(\int_{0}^{t} e d t\right)^{T} K_{D} \dot{e}-\left(\int_{0}^{t} e d t\right)^{T} K_{I}\left(\int_{0}^{t} e d t\right) .
\end{aligned}
$$

Substituting (16) and (17) into (15) yields

$$
\dot{V}=-\dot{e}^{T} K_{D} \dot{e}-\gamma e^{T} K_{P} e-\left(\int_{0}^{t} e d t\right)^{T} K_{I}\left(\int_{0}^{t} e d t\right)-\dot{e}^{T}\left(K_{D}+K_{I}\right)\left(\int_{0}^{t} e d t\right)+1 / 2 x^{T} \dot{M}(q) x-x^{T} C(q, \dot{q}) x+
$$

$\tilde{P}^{T} \Phi^{-1} \dot{\tilde{P}}+x^{T} \Psi\left(q, \dot{q}, \dot{q}_{k}, \ddot{q}_{k}\right) \tilde{P}+x^{T}(V-u)$

With property (P2) one obtains

$1 / 2 x^{T} \dot{M}(q) x-x^{T} C(q, \dot{q}) x=1 / 2 x^{T}[\dot{M}(q)-2 C(q, \dot{q})] x=0$

Note that

$x^{T} \Psi\left(q, \dot{q}, \dot{q}_{k}, \ddot{q}_{k}\right) \tilde{P}=\tilde{P}^{T} \Psi^{T}\left(q, \dot{q}, \dot{q}_{k}, \ddot{q}_{k}\right) x, \quad \dot{\tilde{P}}=\dot{\hat{P}}$

And one gets

$\tilde{P}^{T} \Phi^{-1} \dot{\tilde{P}}+x^{T} \Psi\left(q, \dot{q}, \dot{q}_{k}, \ddot{q}_{k}\right) \tilde{P}=\tilde{P}^{T} \Phi^{-1}\left[-\Phi \Psi^{T}\left(q, \dot{q}, \dot{q}_{k}, \ddot{q}_{k}\right) x\right]+\tilde{P}^{T} \Psi^{T}\left(q, \dot{q}, \dot{q}_{k}, \ddot{q}_{k}\right) x=0$

Thus, one obtains

$\dot{V}=-\dot{e}^{T} K_{D} \dot{e}-\gamma e^{T} K_{P} e-\left(\int_{0}^{t} e d t\right)^{T} K_{I}\left(\int_{0}^{t} e d t\right)-\dot{e}^{T}\left(K_{D}+K_{I}\right)\left(\int_{0}^{t} e d t\right)+x^{T}(V-u)$

Note that the following equalities and inequalities hold

$$
\begin{gathered}
\dot{e}^{T} K_{D} \dot{e}=\sum_{i=1}^{n} K_{D i} \dot{e}_{i}^{2}, \quad \gamma e^{T} K_{P} e=\sum_{i=1}^{n} \gamma K_{P i} e_{i}^{2}, \quad\left(\int_{0}^{t} e d t\right)^{T} K_{I}\left(\int_{0}^{t} e d t\right) \\
=\sum_{i=1}^{n} K_{i 1}\left(\int_{0}^{t} e d t\right)^{2} \\
\dot{e}^{T}\left(K_{D}+K_{I}\right)\left(\int_{0}^{t} e d t\right)=\sum_{i=1}^{n}\left(K_{D i}+K_{I i}\right) \dot{e}_{i}\left(\int_{0}^{t} \dot{e}_{i} d t\right) \\
-\sum_{i=1}^{n}\left(K_{D i}+K_{I i}\right) \dot{e}_{i}\left(\int_{0}^{t} e_{i} d t\right) \leq 1 / 2 \sum_{i=1}^{n}\left(K_{D i}+K_{I i}\right)\left[\dot{e}_{i}^{2}+\left(\int_{0}^{t} e_{i} d t\right)^{2}\right]
\end{gathered}
$$

Hence, one gets

$$
\dot{V}=-\sum_{i=1}^{n} K_{D i} \dot{e}_{i}^{2}-\sum_{i=1}^{n} \gamma K_{P i} e_{i}^{2}-\sum_{i=1}^{n} K_{I i}\left(\int_{0}^{t} e_{i}\right)^{2}-\sum_{i=1}^{n}\left(K_{D i}+K_{I i}\right) \dot{e}_{i}\left(\int_{0}^{t} e_{i} d t\right)+x^{T}(V-u)
$$

Substituting from (23) into the previous equation

$$
\begin{aligned}
& \dot{V} \leq-\sum_{i=1}^{n} K_{D i} \dot{e}_{i}^{2}-\sum_{i=1}^{n} \gamma K_{P i} e_{i}^{2}-\sum_{i=1}^{n} K_{I i}\left(\int_{0}^{t} e_{i} d t\right)^{2}+1 / 2 \sum_{i=1}^{n}\left(K_{D i}+K_{I i}\right)\left[\dot{e}_{i}^{2}+\left(\int_{0}^{t} e_{i} d t\right)^{2}\right]+x^{T}(V-u) \\
& \dot{V} \leq-\sum_{i=1}^{n}\left[K_{D i}-1 / 2\left(K_{D i}+K_{I i}\right)\right] \dot{e}_{i}^{2}-\sum_{i=1}^{n} \gamma K_{P i} e_{i}^{2}-\sum_{i=1}^{n}\left[K_{I i}-1 / 2\left(K_{D i}+K_{I i}\right)\right]\left(\int_{0}^{t} e_{i} d t\right)^{2}+x^{T}(V-u)
\end{aligned}
$$

Now considering the term of $x^{T}(V-u)$, 


$$
\begin{aligned}
x^{T} V & =\sum_{i=1}^{n} x_{i}\left[-\left(b_{1}+b_{2}\|e\|+b_{3}\|\dot{e}\|+b_{4}\left\|\int_{0}^{t} e d t\right\|\right) \operatorname{sgn}\left(x_{i}\right)\right] \\
& =\sum_{i=1}^{n}\left[-\left(b_{1}+b_{2}\|e\|+b_{3}\|\dot{e}\|+b_{4}\left\|\int_{0}^{t} e d t\right\|\right)\left|x_{i}\right|\right]
\end{aligned}
$$

$\leq \sum_{[=1}^{n}\left(-\|u\| .\left|x_{i}\right|\right)$

Hence the assumption (A2) has been used. Not that

$-x^{T} u \leq\left\|x^{T}\right\| .\|u\|$.

Defining $\left\|x^{T}\right\|=\sum_{i=1}^{n}\left|x_{i}\right|$ gives

$$
\begin{aligned}
\dot{V} \leq-\sum_{i=1}^{n}\left[K_{D i}-\right. & \left.1 / 2\left(K_{D i}+K_{I i}\right)\right] \dot{e}_{i}^{2}-\sum_{i=1}^{n} \gamma K_{P i} e_{i}^{2}-\sum_{i=1}^{n}\left[K_{I i}-1 / 2\left(K_{D i}+K_{I i}\right)\right]\left(\int_{0}^{t} e_{i} d t\right)^{2} \\
& +\sum_{i=1}^{n}\left(-\|u\| .\left|x_{i}\right|\right)+\left\|x^{T}\right\| .\|u\|
\end{aligned}
$$

$\dot{V} \leq-\sum_{i=1}^{n}\left[K_{D i}-1 / 2\left(K_{D i}+K_{I i}\right)\right] \dot{e}_{i}^{2}-\sum_{i=1}^{n} \gamma K_{P i} e_{i}^{2}-\sum_{i=1}^{n}\left[K_{I i}-1 / 2\left(K_{D i}+K_{I i}\right)\right]\left(\int_{0}^{t} e_{i} d t\right)^{2}$

With $K_{D i}=K_{I i}>0$ and $K_{P i}>0$ oneobtains

$K_{D i}-1 / 2\left(K_{D i}+K_{I i}\right)=0, \quad \gamma K_{P i}>0, \quad K_{I i}-1 / 2\left(K_{D i}+K_{I i}\right)=0$

Finally, one gets

$\dot{V} \leq-\sum_{i=1}^{n} \gamma K_{P i} e_{i}^{2}$

From the proof and analysis above we know that the function $\dot{V}$ is negative and vanishes if and only if e $(\mathrm{t})=0$; thus the position tracking error goes to zero as time goes to infinity; namely, $\lim _{t \rightarrow \infty} e(t)=0$.

According to the assumption (A3) we obtain that $\dot{e}(t)$ : is uniformly continuous [35]. Consider the following formula holds:

$\lim _{t \rightarrow \infty} \int_{0}^{t} \dot{e}(\tau) d \tau=\lim _{t \rightarrow \infty}[e(t)-e(0)]=-e(0)<\infty$

which implies that the $\operatorname{limit}_{t \rightarrow \infty} \int_{0}^{t} \dot{e}(\tau) d \tau$ is existent and bounded. Therefore, it follows from the Barbalat's lemma [36] that $\dot{e}(t) \rightarrow 0$, ast $\rightarrow \infty$; thatis, $\lim _{t \rightarrow \infty} \dot{e}(t)=0$.

Hence, the designed controller can guarantee the equilibrium $(\mathrm{e}, \dot{e})=(0,0)$ globally asymptotically stable. It is also seen that the parameter vector $\widetilde{P}$ is bounded but does not necessarily converge to zero.

\section{SIMULATION STUDY}

In order to illustrate the performance of the proposed robust adaptive PID controller, simulation study has been carried out using VisSim language. The simulation model is a two-degree of freedom flexible joint robot (FJR) manipulator with rotary joints. According to (1), its inertia matrix, coriolos and centrifugal vector and gravity vector can be described as follows [32].

$\mathrm{M}(\mathrm{q}) \quad=\left[\begin{array}{cc}d_{1}+2 d_{2} \cos \left(q_{2}\right) & d_{3}+d_{2} \cos \left(q_{2}\right) \\ d_{3}+d_{2} \cos \left(q_{2}\right) & d_{3}\end{array}\right]$

$\mathrm{C}(\mathrm{q}, \dot{q})=\left[\begin{array}{c}-\dot{q}_{2}\left(2 \dot{q}_{1}+\dot{q}_{2}\right) \sin \left(q_{2}\right) \\ \dot{q}_{2}^{2} d_{2} \sin \left(q_{2}\right)\end{array}\right] \quad G\left(q_{1}, q_{2}\right)=\left[\begin{array}{c}\left(m_{1}+m_{2}\right) r_{1} \cos q_{2}+m_{2} r_{2} \cos \left(q_{1}+q_{2}\right) \\ m_{2} r_{2} \cos \left(q_{1}+q_{2}\right)\end{array}\right]$

Where

$d_{1}=I_{1}+I_{2}+a_{1}^{2} m_{1}+l_{1}^{2} m_{r 2}+m_{2}\left(l_{1}^{2}+a_{2}^{2}\right)$ 


$$
\begin{gathered}
d_{2}=2 m_{2} l_{1} a_{2} \\
d_{3}=l_{2}+a_{2}^{2} m_{2}
\end{gathered}
$$

Where $\mathrm{I}_{1}, \mathrm{I}_{2}, \mathrm{~m}_{1}, \mathrm{~m}_{2}, \mathrm{a}_{1}, \mathrm{a}_{2}, \mathrm{l}_{1}, \mathrm{l}_{2}$, and $\mathrm{m}_{\mathrm{r} 2}$ are the moment of inertia about an axis parallel to the axis of rotation passing through the center of mass, the mass, the distance from the center of rotation to the center of mass, length of the first and second link, respectively, and the mass of the second rotor.

At first, linearize (1) and give the parameter matrix $\Psi$ and P. According to (5) the following equality holds:

$M(q) \ddot{q}_{k}+C(q, \dot{q}) \dot{q}_{k}+G(q)=\Psi\left(q, \dot{q}, \dot{q}_{k}, \ddot{q}_{k}\right) P$

Let the matrices $\Psi$ and $P$ have the following forms:

$\Psi=\left[\begin{array}{lll}\Psi_{11} & \Psi_{12} & \Psi_{13} \\ \Psi_{21} & \Psi_{22} & \Psi_{23}\end{array}\right]$

Then the parameters can be derived as follows:

$$
\begin{gathered}
\Psi_{11}=\ddot{q}_{i k}+\left(g / r_{1}\right) \cos q_{2}, \quad \Psi_{12}=\ddot{q}_{1 k}+\ddot{q}_{2 k}, \quad \Psi_{21}=0, \quad \Psi_{22}=\Psi_{12} \\
\Psi_{13}=2 \ddot{q}_{1 k} \cos q_{2}+\ddot{q}_{2 k} \cos q_{2}-\dot{q}_{2} \dot{q}_{1 k} \sin q_{2}-\left(\dot{q}_{1}+\dot{q}_{2}\right) \dot{q}_{2 k} \sin q_{2}+\left(g / r_{1}\right) \cos \left(q_{1}+q_{2}\right) \quad \text { (33) } \\
\Psi_{23}=\dot{q}_{1} \dot{q}_{1 k} \sin q_{2}+\ddot{q}_{1 k} \cos q_{2}+\left(g / r_{1}\right) \cos \left(q_{1}+q_{2}\right) \\
P_{1}=\left(m_{1}+m_{2}\right) r_{1}^{2}, \quad P_{2}=m_{2} r_{2}^{2}, \quad P_{3}=m_{2} r_{1} r_{2}
\end{gathered}
$$

The link parameters of the robot manipulator are given as listed in table 1[34]. The upper bound parameters are selected as $b_{1}=2, b_{2}=3, b_{3}=6$, and $b_{4}=1$. The disturbances vector is chosen as $u=\left[\begin{array}{ll}1.0 & 1.0\end{array}\right]^{T}+2 e+5 \dot{e}$. The reference trajectories are $q_{1 d}=\cos (\pi t), \quad q_{1 d}=\cos (\pi t)$. The initial values of manipulator positions and velocities are selected as $\left[q_{1} \dot{q}_{1} q_{2} \dot{q}_{2}\right]^{T}=\left[\begin{array}{llll}0.6 & 0 & 0.3 & 0\end{array}\right]^{T}$. Take the gain coefficients of the robust adaptive PID control as:

$K_{P}=\operatorname{diag}[1000,400], K_{D}=\operatorname{diag}[180,150], K_{I}=K_{D}, \gamma=5, \Phi=\operatorname{diag}[5.0,5.0,5.0\}$

The adaptive PD control gains $K_{P}$ and $K_{D}$ are determined by [26,27].

\section{RESULTS AND DISCUSSION}

In order to show the advantage of the proposed control law, a simulations studies are carried out. Adopting the robust PID adaptive control law described in (7-10) and (32-34) to simulate the tracking effects of robot manipulator, the simulation results can be seen from Figures 2,3,4,5, and 6. These figures indicate that the robust adaptive PID control scheme can compensate the bounded external disturbances and guarantee the manipulator systems to track the desired position and velocity trajectories accurately with quit small tracking errors in finite time. The adaptive algorithm can effectively estimate the unknown constant vector $\mathrm{P}$ which describes the mass characteristics of robot manipulator.

The robust adaptive PID controller can provide better control performance due to the incorporation of an integral action within both the PID control and robust adaptive algorithm.

PID control term, it exhibits the superiority in contrast to the PD one in the adaptive PD controllers \{26,27]. In the case of PD control, the differential element has no effect on steady-state process, although the steady-state errors can be smaller by increasing the proportional gain which may cause the system to be unstable. In addition, the differential action is very sensitive to system noise. While the PID control includes an integral element which can rise the indiscrimination degree of the system, so it can enhance the steady-state performance of the closed -loop system. Also, the PID control can provide one negative real zero, which contributes to improve the dynamic performance of robot manipulator systems.

Adaptation algorithm term, in this work an integral element acts on the estimation law $\dot{\hat{P}}$ via the variable $\mathrm{x}$ (see (3) and (10)), note that continuously accumulated position errors under the action of integration element results in a bigger value of $\dot{\hat{P}}$ than that of $\{26,27]$, which implies that the estimated rate of $\dot{\hat{P}}$ is increased. As a consequence, the dynamic performance and the finite-time estimation accuracy of the estimate vector $\dot{\hat{P}}$ are improved. In addition to these, assumption (A2) provides a bigger upper bound of the external disturbances than the one which is defined as $\|u\| \leq b_{1}+b_{2}\|e\|+b_{3}\|\dot{e}\|$ in $[26,27]$. However, the enlarged external disturbances can be compensated by the enhanced robust term V (see(3),(9)). It should emphasize that, the dynamic performance of the closed-loop system can be improved in the whole process by adopting the robust adaptive PID control law.

Simulation results verify the advantages of the proposed control scheme. The simulation curves Figures 2,3,4, and 5 show that the robust adaptive PID controller provide higher accuracy in finite-time position and velocity tracking control, moreover it results in better dynamic performance of the manipulator systems. Observing the control input curves Figures 6a, and 6b, we can see that the proposed control law leads to less chattering effect, which is beneficial to prolong the service life of robot. Also, it can be verified that the proposed control law can ensure a faster convergence rate and smaller overshoot of the system states tracking to 
the desired trajectories. From the previous analysis, it is clear that this work presents an effective control method for robot manipulator under bounded external disturbances.

\section{CONCLUSION}

This paper describes the design and simulation implementation of a new robust adaptive PID controller for trajectory tracking control of a two-link flexible joints robotmanipulator with known upper bound of the external disturbances. The main feature of this design is that it combines PID control law with robust adaptive algorithm. The adaptive algorithm is utilized to estimate the unknown constant vector $P$ online, while the robust adaptive term and the PID control are used to cope with the bounded external disturbances, adaptive approximation errors, and trajectory tracking errors.

The convergence and stability properties of the closed-loop system are guaranteed, the tolerable external disturbances are enlarged, the dynamic performance of the manipulator systems is improved, and the finite-time tracking control accuracy is enhanced by adopting the proposed control scheme. Simulation results show the advantages of the designed controller and verify that it is able to cope with the external disturbances arises in practical control, and uncertain constant parameters in system dynamic.

\section{REFERENCES}

[1] Ozgoli S. and Taghirad H. D., "A survey on the control of flexible joint robots", Asian Journal of Control, Vol. 8, No. 4, pp.1-15, December 2006.

[2] Koivo A. J., and GUO T.H., "Adaptive linear controller for robotic manipulator", IEEE Trans., 1984, AC-28, pp. 162-171.

[3] SINGH S.N., "Control and stabilization of a nonlinear uncertain elastic robotic arm", IEEE Trans., 1988, AES-24, pp. 114-123.

[4] KIM G.K., and SHIN K.G., "An adaptive model following control of industrial manipulator", IEEE Trans., 1983, AES-19, pp. 805-814.

[5] SINGH S.N., and SCHY A.A., "Robust trajectory following control of robotic system", j. Dynamic systems, Measurement and Control, 1985, 107, pp. 308-315.

[6] HA I.J., and GILBERT E.G., "Roust tracking in nonlinear systems", IEEE Trans., 1987, AC-32, pp. 763-771.

[7] SINGH S.N., and SCHY A.A., "Control of elastic robotic system by nonlinear inversion and model damping", j. Dynamic Systems, Measurement, and Control, 1986, 108, pp. 180- 189.

[8] YOUNG K.D., "A variable structure model following control design for robotics applications", IEEE Trans., 1988, RA-4, pp.556=561.

[9] SINGH S.N., and ZAKHARIA Y.N., "Variable structure control of a robotic arm in the presence of uncertainty", j. Robotic System, 1989, 6, pp. 111-132.

[10] YEUNG K.S., and CHEN Y.P., "A new controller design for manipulators using the theory of variable structure systems", IEEE Trans., 1988, AC-33, pp.200-206.

[11] HASHIMOTO H., MARUYAMA K., and HARASHIMA, F., "A microprocessor-based robot manipulator control with sliding mode", IEEE Trans., 1987, IE-34, pp. 11-18.

[12] Kelly R., Carelli R.,"A class of nonlinear PD-type controllers for robot manipulators", Journal of Robotic Systems, Vol. 13, no. 12, pp. 793-802, 1996.

[13] Kawamura S., Miyazaki F., and Arimoto,"Is a local PD feedback control law effective for trajectory tracking of robot motion?", Proceedings of the IEEE International Conference on Robotics and Automation, Vol. 3, pp. 1335-1340, 1988.

[14] Arimoto S., and Miyazaki F.," Stability and robustness of PID feedback control for robot manipulators of sensory capability", Proceeding of the $1^{\text {st }}$ International Symposium on Robotics Research, pp. 783-799, 1984.

[15] Jafarov E. M., Parlakci M. N. A., and Istefanopulos Y.,"A new variable structure PID-controller design for robot manipulators", IEEE Transaction on Control Systems Technology, Vol. 13, no. 1, pp. 122-130, 2005.

[16] Leitmann G. ,"On the efficacy of nonlinear control in uncertain linear systems", Journal of Dynamic Systems, Measurement and Control, Vol. 103, no. 2, pp. 95-102, 1981.

[17] Corless M. J. and Leitmann G., "Continuous state feedback guaranteeing uniform ultimate boundedness for uncertain dynamic systems", Institute of Electrical and Electronics Engineers, Vol. 26, no. 5, pp. 1139-1144, 1981.

[18] Spong M. W., "On the robust control of robot manipulators", Institute of Electrical and Electronics Engineers, Vol. 37, no. 11, pp. 1782-1786, 1992.

[19] Yaz E., "Commons on "on the robust control of robot manipulators" by Spong M.W", IEEE Transactions on Automatic Control, Vol. 38, no. 3, pp. 511-512, 1993. 
[20] Koo K. M., and Kim J. H., "Robust control of robot manipulator with parametric uncertainty", Institute of Electrical and Electronic Engineerrs, Vol.39, no. 6, pp. 1230-1233, 1994.

[21] Danesh M., Keshmiri M., and Sheikholeslam F., "Developing a robot control scheme robust to uncertain model parameters and unmodeled dynamics", Proceeding of the $1^{\text {st }}$ IEEE Conference on Industrial Electronics and Applications (ICIEA '06), pp. 1-6, May 2006.

[22] Lim K. Y., and Eslami M., "Robust adaptive controller designs for robot manipulator systems", IEEE Journal of Robotics and Automation, Vol. 3, no. 1, pp. 54-66, 1987.

[23] Johasson R., "Adaptive control of robot manipulator motion", IEEE Transactions on Robotics and Automation, Vol. 6, no. 4, pp.483-490, 1990.

[24] Yang Z. J., Fukushima Y., and Qin P., "Decentralized adaptive robust control of robot manipulators using disturbance observers", IEEE Transactions on Control Systems Technology, Vol. 20, no. 5, pp. 1357-1365, 2012.

[25] Fateh M. M., and Khorashadizadeh S., "Robust control of electrically driven robots by adaptive fuzzy estimation of uncertainty", Nonlinear Dynamics, Vol. 69, no. 3, pp. 1465-1477, 2012.

[26] Bai H. J., Tang D. F., and Cao J. B., "A robust adaptive PD control method of robot manipulator", Journal of Mechanical Transmission, Vol. 35, no. 11, pp. 34-40, 2011.

[27] Ge D., and Jiang S., "Self-adaptivePD control of robot manipulator", Proceedings of the $8^{\text {th }}$ ACIS International Conference on Software Engineering, Artificial Intelligence, Networking, and Parallel/Distributed Computing (SNPD '07), pp. 605-610, August 2007.

[28] Fateh M.M., "Robust control of Flexible-joint robots using voltage control strategy", J. of Nonlinear Dynamics, Vol. 67, Issue 2, pp. 1525-1537, Jan. 2012.

[29] Artemisk K., Zoe D. and George A.R., "Prescribed performance tracking for flexible joint robot with unknown dynamics and variable elasticity", Automatica, Vol. 49, Issue 5, pp. 1137-1147, May 2013.

[30] Ulrich S., Sasiadek J.Z., and Barkana I., "Modeling and direct adaptive control of flexible joint manipulator", J. of Guidance, control and Dynamic, Vol. 35, No. 1, pp.25-39, 2012.

[31] Yongming LI., Shaocheng T. and Tieshan LI., "Adaptive fuzzy output feedback control for a single link flexible robot manipulator driven DC motor Via backstepping", Nonlinear Analysis Applications, Vol. 14, Issue 1, pp. 483-494, Feb. 2013.

[32] Spong M., "Modeling and control of elastic joint robots", J. of Dyn. Syst. Meas. Cont., Vol. 109, No.4, pp. 310-319, December 1987.

[33] Spong M. and Vidyasagar M.W., "Robot dynamics and control", John Wiley \& Sons, New York, USA, Inc. 1989.

[34] Massoud A. and Elmaraghy H., "Design, dynamics, and identification of a flexible joint robot manipulator", Proc. Of the IASTED Conf. on Robotics and Manuf. Oxford, England, pp. 72-75, Sept.1993.

[35] Slotine J., and Li W., Applied Nonlinear Control, Prentice Hall, New Jersey, NJ, USA, 1991.

[36] Khalil H. K, Nonlinear Systems, Prentice Hall, Upper Saddle River, USA, $3^{\text {rd }}$ edition, 2003.

Table 1. Robot parameter from design and Sin Sweep Identification

\begin{tabular}{|c|c|c|c|c|c|c|}
\hline & $\begin{array}{c}\mathbf{J}_{11} \\
\left(\mathbf{K g} . \mathrm{m}^{2}\right)\end{array}$ & $\begin{array}{c}\mathbf{J}_{12} \\
\left(\mathbf{K g} . \mathrm{m}^{2}\right)\end{array}$ & $\begin{array}{c}\mathbf{d}_{1} \\
\left(\mathbf{K g} . \mathrm{m}^{2}\right)\end{array}$ & $\begin{array}{c}\mathbf{d}_{2} \\
\left(\mathbf{K g} . \mathrm{m}^{2}\right)\end{array}$ & $\begin{array}{c}\mathbf{b}_{1} \\
(\text { N.m.s/rad })\end{array}$ & $\begin{array}{c}\mathbf{b}_{2} \\
(\text { N.m.s/rad })\end{array}$ \\
\hline Sin Sweep & & & $\mathbf{2 . 0 8 7}$ & $\mathbf{0 . 2 1 6}$ & $\mathbf{2 . 0 4 1}$ & $\mathbf{0 . 2 4 2}$ \\
\hline I - DEAS & $\mathbf{0 . 2 2 6 9}$ & $\mathbf{0 . 0 4 2 9}$ & $\mathbf{2 . 1 1 0}$ & $\mathbf{0 . 2 2 3}$ & & \\
\hline
\end{tabular}

\begin{tabular}{|c|c|c|c|c|c|c|}
\hline & $\begin{array}{c}\mathbf{b}_{\mathbf{m} 1} \\
(\mathbf{N} . m . s / \mathbf{r a d})\end{array}$ & $\begin{array}{c}\mathbf{b}_{\mathbf{m} 2} \\
(\mathbf{N} . \mathbf{m} . \mathbf{S} / \mathbf{r a d})\end{array}$ & $\begin{array}{c}\mathbf{k}_{1} \\
(\mathbf{N} . \mathbf{m} / \mathbf{r a d})\end{array}$ & $\begin{array}{c}\mathbf{k}_{2} \\
(\mathbf{N} . \mathbf{m} / \mathbf{r a d})\end{array}$ & $\begin{array}{c}\mathbf{J}_{\mathbf{m} 1} \\
\left(\mathbf{K g} . \mathbf{m}^{2}\right)\end{array}$ & $\begin{array}{c}\mathbf{J}_{\mathbf{m} 2} \\
\left(\mathbf{K g} . \mathbf{m}^{2}\right)\end{array}$ \\
\hline Sin Sweep & $\mathbf{1 . 2 5 4}$ & $\mathbf{0 . 1 1 9}$ & $\mathbf{1 2 5 . 5 6}$ & $\mathbf{3 1 . 2 7}$ & $\mathbf{0 . 1 2 2 4}$ & $\mathbf{0 . 0 1 6 8}$ \\
\hline I - DEAS & & & $\mathbf{1 9 8 . 4 9}$ & $\mathbf{5 1 . 1 1}$ & $\mathbf{0 . 1 2 2 6}$ & $\mathbf{0 . 0 1 7}$ \\
\hline
\end{tabular}


Fig.1 Framework of control law with known upper bound of the disturbances.
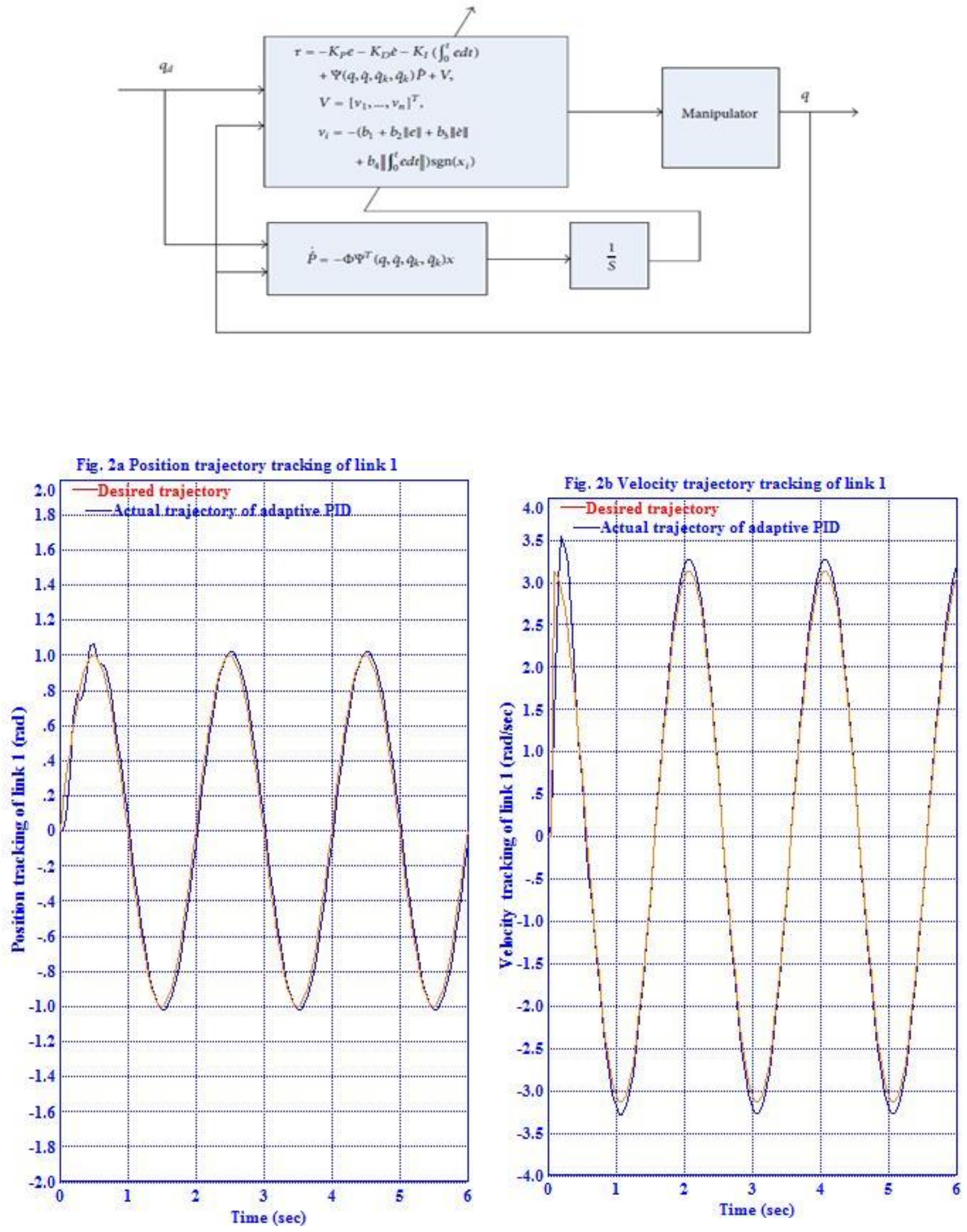
Fig, 3a Position trajectory tracking error of link 1

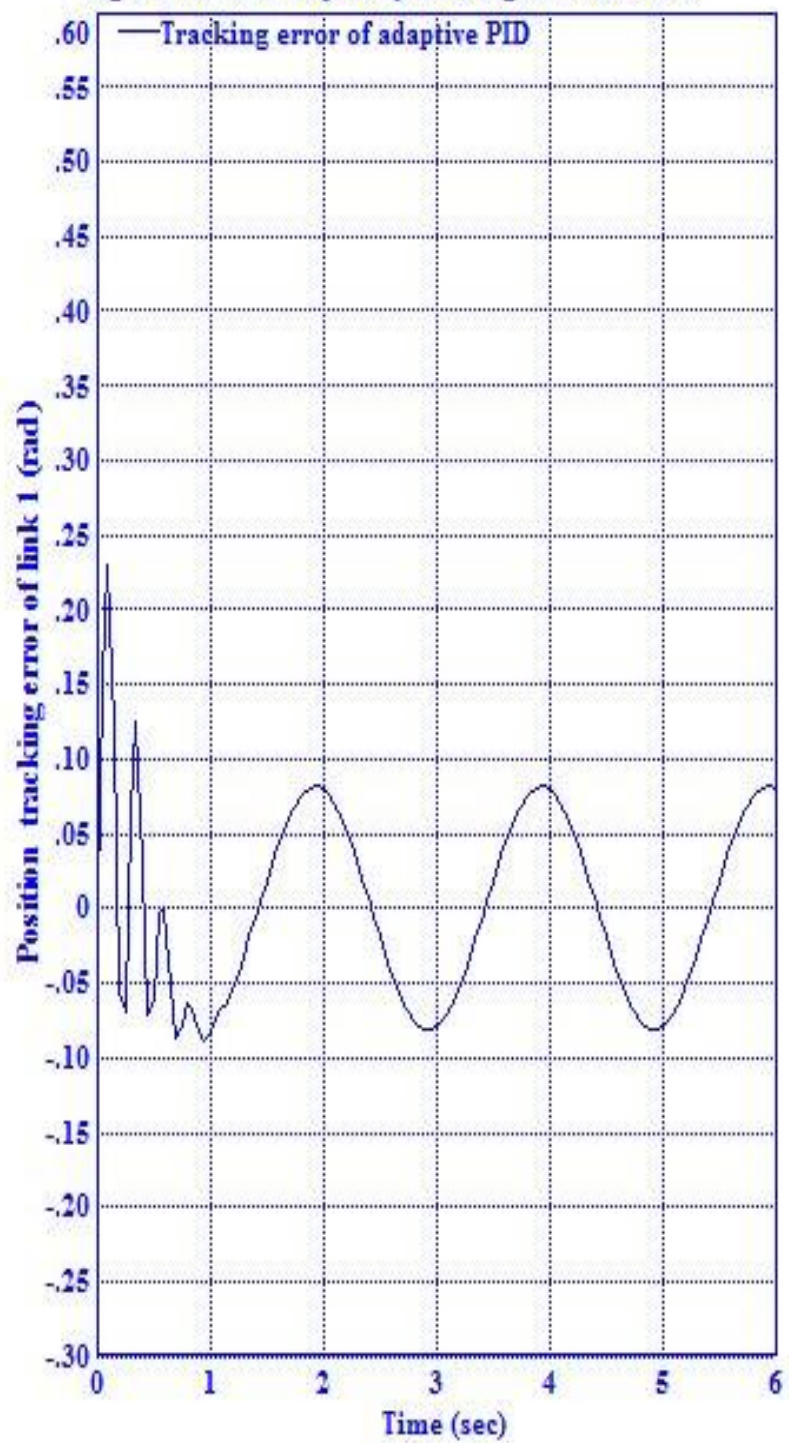

Fig. 3b Velocity trajectory tracking error of link 1

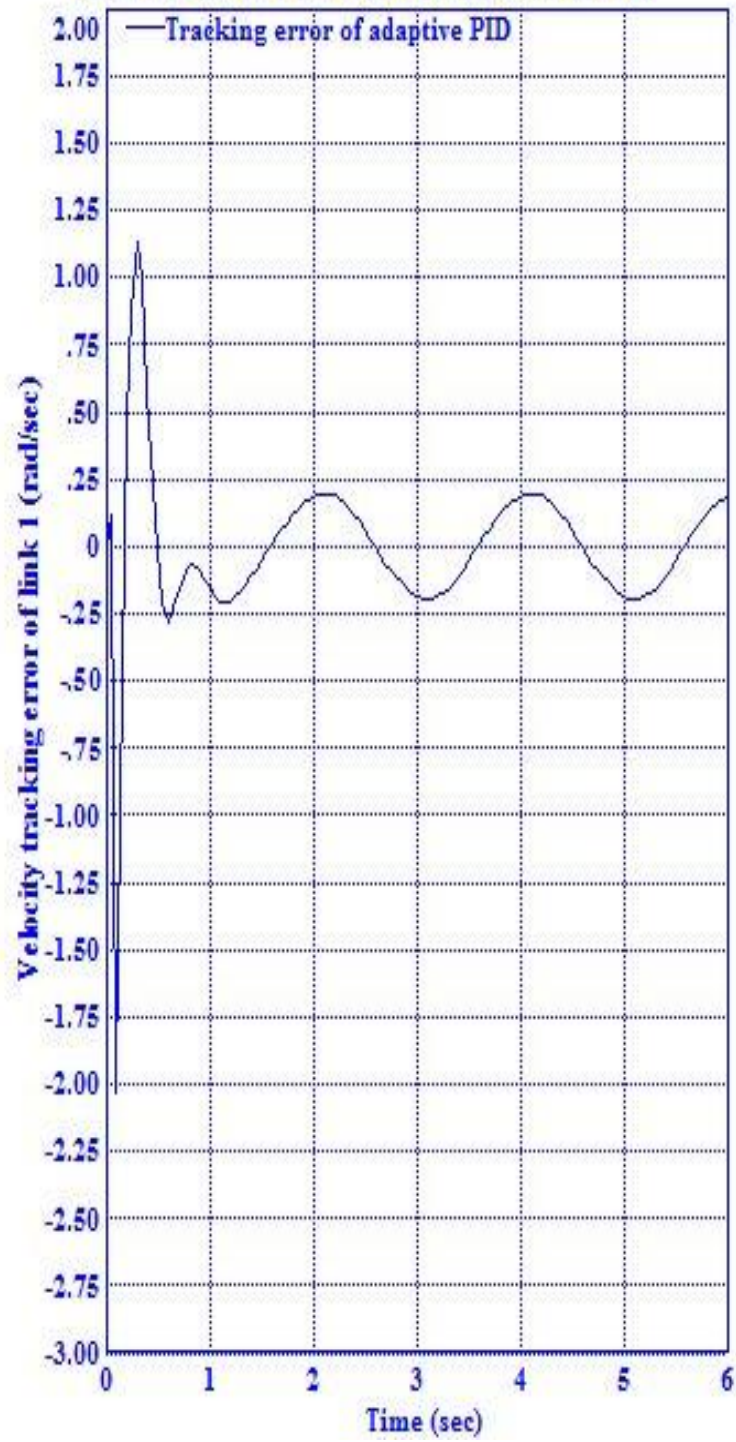


Fig. 4a Position trajectory tracking of link 2

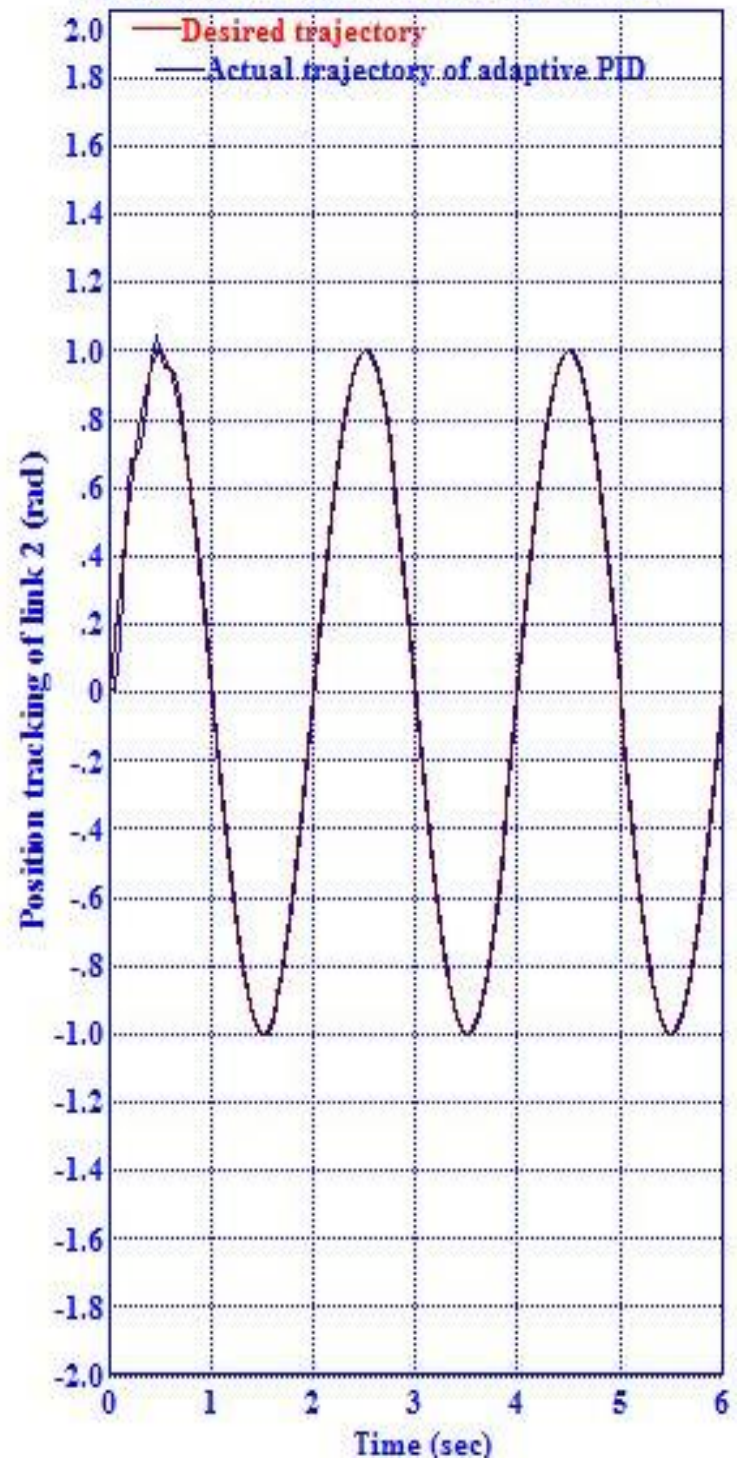

Fig, 4b Velocity trajectory tracking of link 2

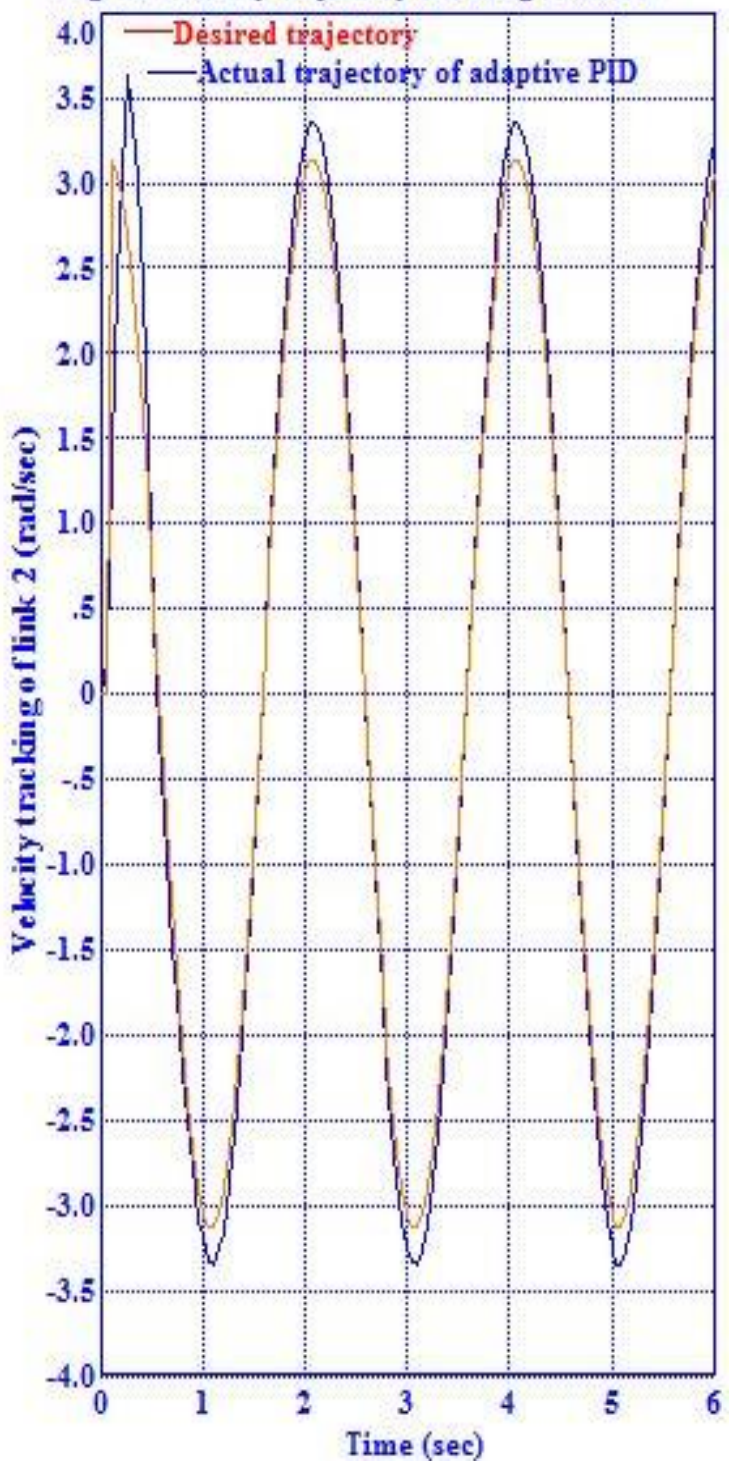


Fig. 5a Position trajectory tracking error of link 2

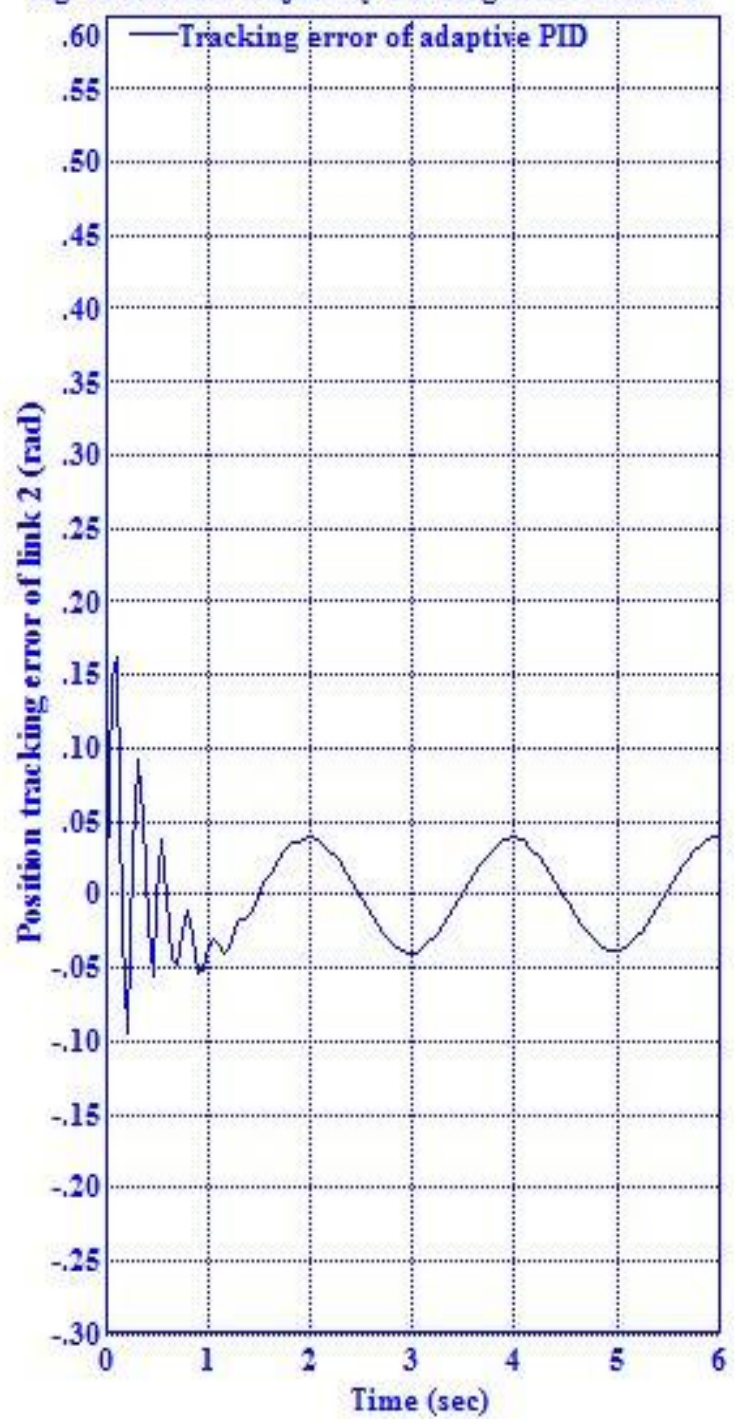

Fig. 5b Velocity trajectory tracking error of link 2

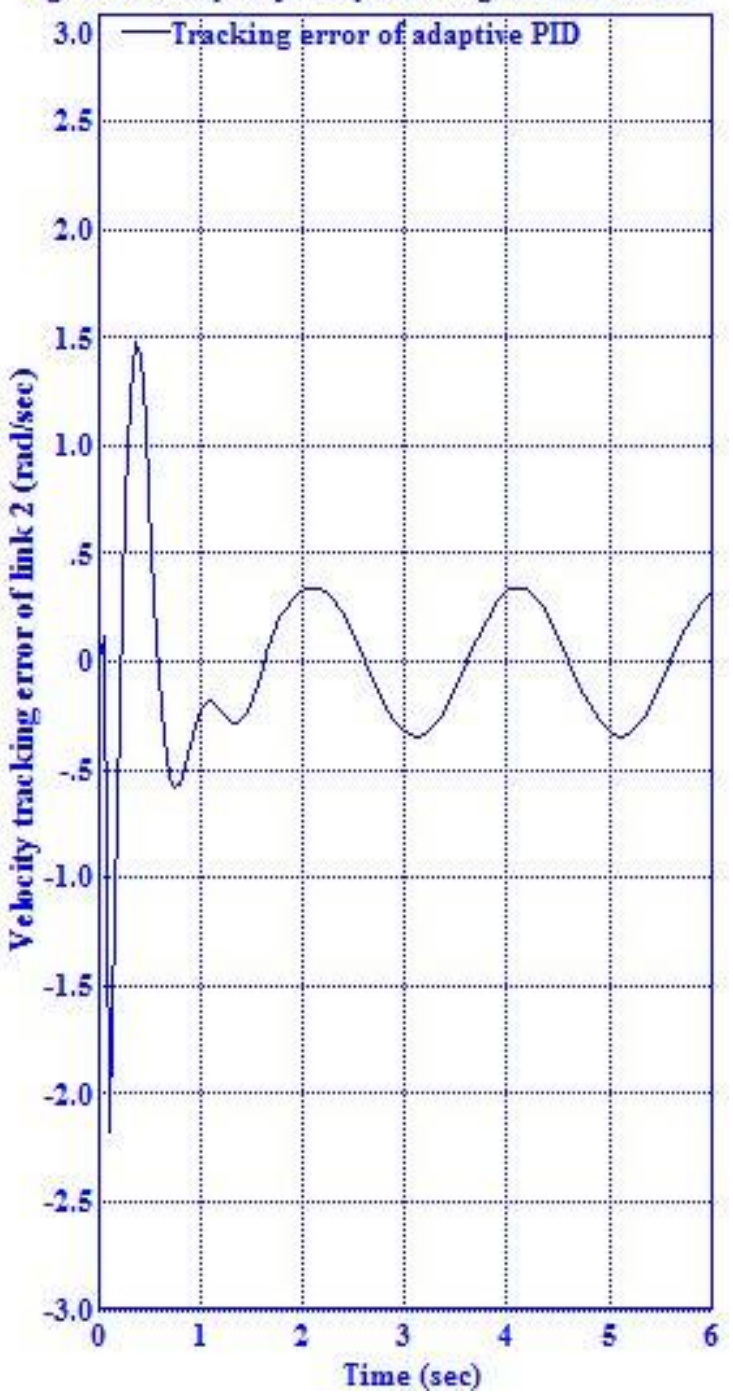


Fig, 6a Control input of link 1

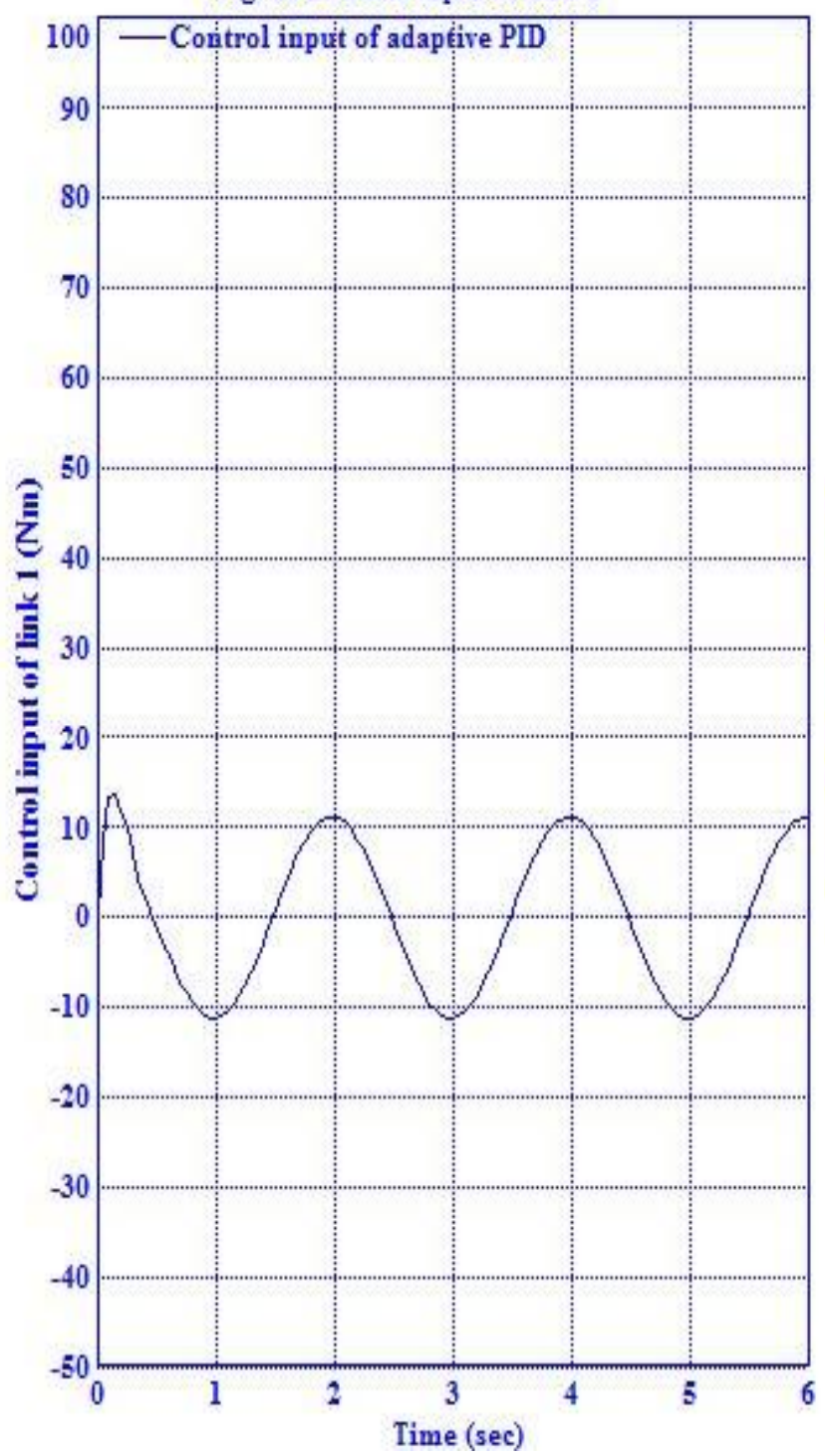

Fig, 6b Control input of link 2

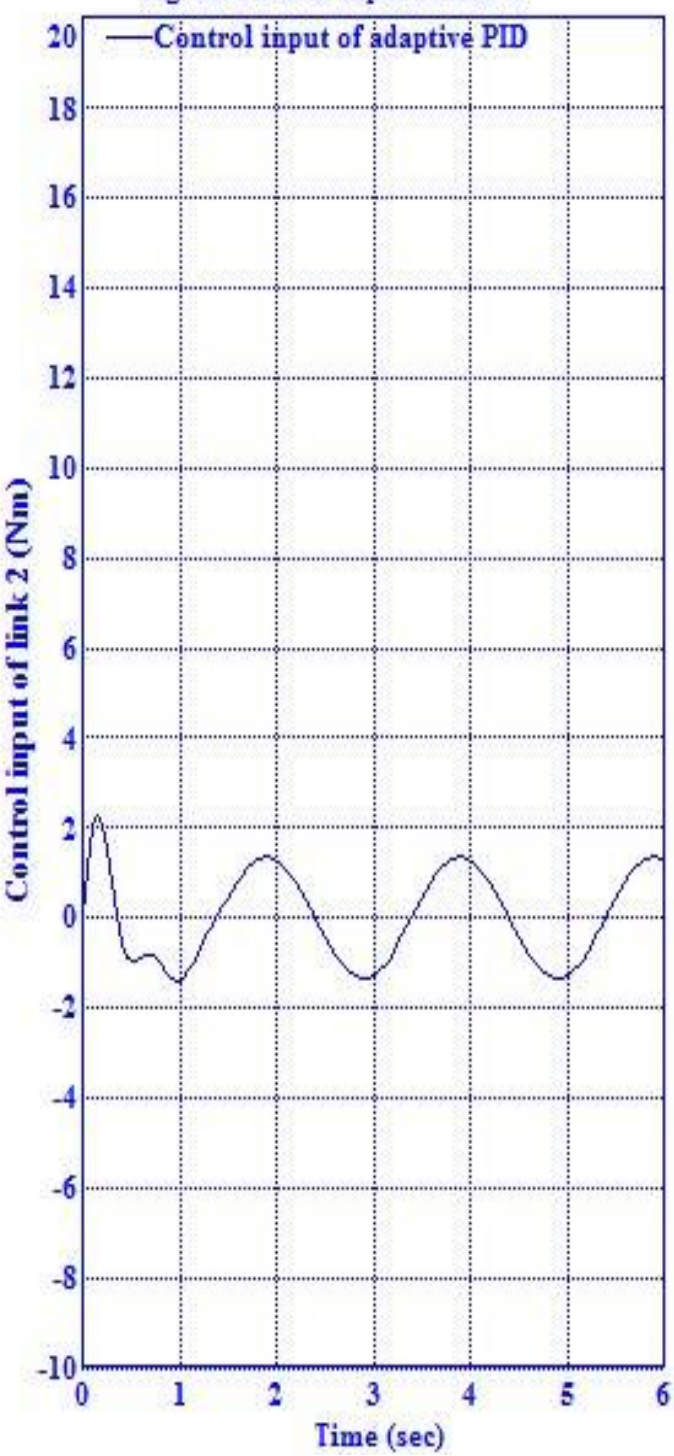

